\title{
Research on the 3-D Reconstruction of Industrial Flame based on the Ratio of Controlled Matching Points
}

\author{
Zhenhua Wei ${ }^{1}$, Limao Ren ${ }^{1}$ and Shibo Song ${ }^{2}$ \\ ${ }^{1}$ North China Electric Power University Control and Computer Engineering \\ Department BeiJing 102206 \\ ${ }^{2}$ Harbin University Math and Science Department Harbin 150080 \\ \{ZhenhuaWei,Limao Ren, Shibo Song,renlimao_910529\}@yeah.net
}

\begin{abstract}
3-D reconstruction of industrial flame could be used to research for the completeness of combustion and flame temperature field. As the flame flicks and has no regular evident characteristic, 3-D reconstruction of flame is difficult and lack of study. The paper proposed a stereo matching algorithm for flame based on distance ratio constraint (DRCFMA). Taking flame characteristics into account and using the processed SIFT operates to get the ratio constraint of matched feature points, the algorithm is applied on the follow-up 3-D reconstruction of flame. The results shows the validity and feasibility of DRCFMA. Compared with conventional algorithms, the proposed algorithm has a better accuracy and shorter time-taken for industrial flame. The experiments had shown that the follow-up 3-D reconstruction of flame has clear and continuously changing greyscale layers.
\end{abstract}

Keywords : flame, 3-D reconstruction, depth map, stereo matching

\section{Introduction}

With the development of industry, the security of boiler furnace in power plant has been paid more and more attention. Along with the rapid improvement of the power plant, the requirement of the boiler furnace safety supervisory system is increasingly high. The furnace flame fully reflects the whole process of industrial combustion. It is also a direct object of observation in combustion. The degree of completeness and stability of combustion are the key points of the power plant monitoring. The result of threedimensional (3-D) reconstruction of furnace flame could be of great help for the followup studies like the flow or the temperature of furnace flame.

As a gas-solid two-phase flow, the flame has following features: its edge is irregular and changes quickly, it flashes at a high frequency, its cross-sectional area is not constant. The above characteristics will lead to the considerable errors when we apply the conventional 3-D reconstruction algorithms to flame. As flame cannot be touched or be marked, the method of artificial point mark cannot be worked, which brings a lot difficulties to 3-D reconstruction of the flame. At present, there are few international teams or individuals involved in the research of 3-D reconstruction of flame. YanYong, the professor at the University of Kent, led the team to make some achievements in the 3$\mathrm{D}$ reconstruction of the flame. They use CT tomographic method for 3-D reconstruction of the flame[1]. As the required equipments are expensive, the method is not easy to be popularised in the industry. Also, the method of CT tomography has a disadvantage that the fewer projections, the greater error[2].

Conventional 3-D reconstruction methods include CT tomography, using discrete and crowded point cloud to represent the surface of model, stereo vision and etc. Most of these methods are applying to the rigid or static objects[3]. In consideration of the 
characteristics of the industrial flame, 3-D reconstruction for flame should be proposed by means of studying and improving the currently existing methods. .

CT tomography equipments are too expensive. The method of dense point cloud takes too much time. The characteristic of flame flicker lead to the poor 3-D reconstruction. The above two disadvantages make that the method of dense point cloud cannot satisfy the requirements of industry flame reconstruction. Flame characteristics determine that the method of stereo vision for 3-D reconstruction of power plant furnace flame is the most convenient and feasible. It is also easy to accept in economic terms. In the process of 3-D reconstruction research, we found that using monocular vision for 3-D reconstruction of flame is undesirable because the flame information that monocular vision get is not comprehensive. Especially when the boundary of flame depth image is discontinuous, there will be burr in 3-D reconstruction result, which lacks sense of reality; Lack of enough global flame information, binocular stereo vision still cannot reach required precision. Multi-view stereo vision will get the real information of flame as much as possible. In view of the flame characteristics, 3-D reconstruction of industrial flame has been studied. A stereo matching algorithm using industrial flame feature points based on multi-view stereo vision is proposed, which is applied to the follow-up 3-D reconstruction of flame. Experiments show that the method of 3-D reconstruction of industrial flame proposed in this paper has reached the real-time and accuracy requirements of the electric power industry.

\section{System Setup}

Two RGB cameras with eight imaging fibre bundles for eight projections have been put around the flame. Each fibre bundles has a total of 30,000 individual optical fibers with a 92 field of view objective lens and arranged on one side of the burner with an angle of $22.5^{\circ}$ between two adjacent fibres. Four of the eight fiber bundles are joined onto a single eyepiece/coupling lens, forming four images onto the same CCD sensor. The optical paths of the eight fiber bundles are passing through the burner central line. Two RGB CCD cameras transmit the image signals to a personal computer by two frame grabbers. The two frame grabbers are synchronized by the supporting computer software, acquire and digitize the image signals and convert them into 2-D matrices. With such an arragement, the image acquisition system produces eight RGB images of the flame simultaneously from eight different directions around the flame.

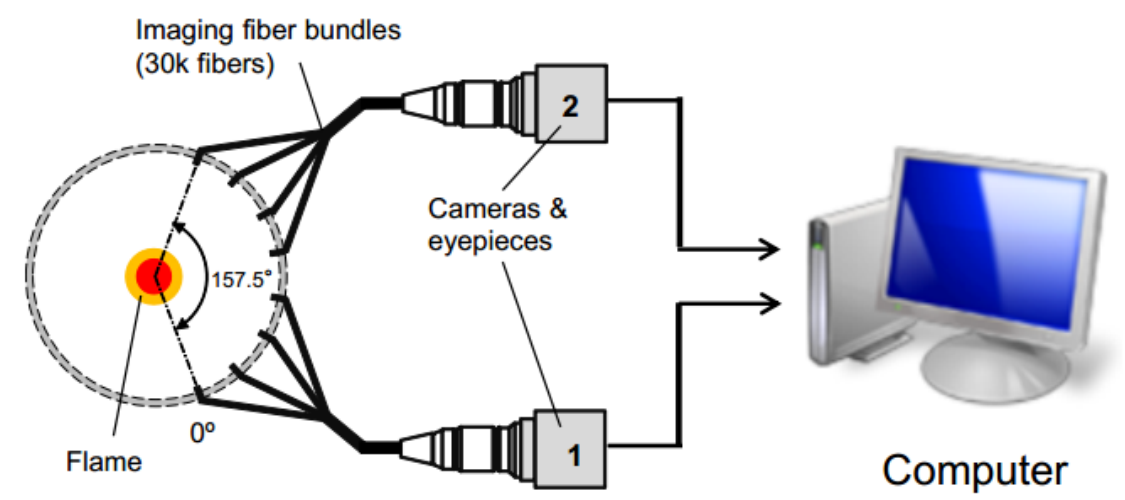

Figure 1. The Flame Image Acquisition System 


\section{SIFT Operator}

Based on the scale space theory, David G.Lowe has proposed to use SIFT operator[4] to describe the local features which could maintain invariance to image scaling, rotation, and affine transformation. SIFT algorithm will detect feature points in scale space and obtain the location and the scale of the key points. Then the main direction of neighborhood gradient of key point is putted as the main direction of key point, in order to realize the independence of scale and direction. SIFT operator can be very helpful to match the same feature points under different shooting angle.

\subsection{SIFT Scale Space}

The core idea of Scale space theory is described in follows : A scale parameter is introduced into the image information processing model. Through continuous changing the scale parameter, different scales of visual processing information are obtained. The essential characteristics of image are extracted by integrating these information. Gaussian convolution kernel is the only linear nuclear could be used for scale conversion. The scale space of 2-D image is defined as (1)

$$
\mathrm{L}(x, y, \sigma)=\mathrm{G}(x, y, \sigma) * \mathrm{I}(x, y)
$$

where $\mathrm{G}(x, y, \sigma)$ is scale variable gaussian function. $\mathrm{I}(x, y)$ represents gray scale images. $\mathrm{L}(x, y, \sigma)$ represents Gaussian Image. $\mathrm{G}(x, y, \sigma)$ is defined as (2)

$$
\mathrm{G}(x, y, \sigma)=\frac{1}{2 \pi \sigma^{2}} e^{-\left(x^{2}+y^{2}\right)} / 2 \sigma^{2}
$$

where $(\mathrm{x}, \mathrm{y})$ is location. $\sigma$ is scale parameter. The bigger $\sigma$ is, the smoother the image will be processed.

Difference of Gaussian scale-space, which is generated by the different scales of gaussian difference nuclear and image convolution, can effectively extract the stable feature points. In gaussian difference scale space, each testing point should be compared with eight adjacent points in the same scale and corresponding $9 * 2$ points in adjacent scales to determine whether or not it is a maximum or minimum value point. In this way, extreme value points can be detected in the scale space and 2-D image space. Extreme value point $\mathrm{D}(x, y, \sigma)$ is defined as (3). The whole of local extreme value points constitute the set of candidate points of SIFT.

$$
\mathrm{D}(x, y, \sigma)=(\mathrm{G}(x, y, k \sigma)-\mathrm{G}(x, y, \sigma)) * \mathrm{I}(x, y)=\mathrm{L}(x, y, k \sigma)-\mathrm{L}(x, y, \sigma)
$$

where $\mathrm{k}$ is the coefficient of scale parameter. Here it was set as $\sqrt{2}$.

\subsection{The Distribution of the Direction of Feature Points}

The direction parameter of each feature point should be specified by using the neighborhood gradient direction distribution features of the feature point, which makes the operator possess rotation invariance.

$$
\begin{aligned}
& \mathrm{m}(\mathrm{x}, y)=\sqrt{(L(x+1, y)-L(x-1, y))^{2}+(L(x, y+1)-L(x, y-1))^{2}} \\
& \theta(\mathrm{x}, y)=\arctan (2 \mathrm{~L}(\mathrm{x}, \mathrm{y}+1)-\mathrm{L}(\mathrm{x}, \mathrm{y}-1)) /(\mathrm{L}(\mathrm{x}+1, \mathrm{y})-\mathrm{L}(\mathrm{x}-1, \mathrm{y}))
\end{aligned}
$$

(4) and (5)are the modulus value and direction of the gradient of the feature point respectively. Where $\mathrm{L}$ is the scale for each feature point respectively.

In study, the sample of neighboring window centered the feature point should be taken and statistical histogram should be drawn to summarize the gradient direction of the neighborhood pixels. The direction of biggest probability unit represents is the principal direction. When another unit exists and its probability is more than $80 \%$, the direction of 
this unit represents the auxiliary direction. A feature point may be specified with multiple direction (one principal direction, more than one auxiliary direction). In that way, the robustness of testing point can be enhanced.

\section{Normalized Cross-Correlation Matching Algorithm}

Normalized Cross-Correlation Matching Algorithm[5] (NCC) is for image matching. A window centered the feature point is opened in the reference image, the matching image set up a sliding window of the same size within the parallax. When the sliding window slide to a particular location, the two windows have the largest gray scale similarity, which means the match is successful.

The schematic diagram of NCC is shown as figure 2.The following are the processes. Firstly, in the reference image (this paper set left image in figure 2 for example), the point $\mathrm{P}\left(x_{i}, y_{j}\right)$ is selected as a match point. Secondly, the template window $\mathrm{W}$ with the size of $\mathrm{N} 1 * \mathrm{~N} 2$ centered pixel coordinates of $\mathrm{P}\left(x_{i}, y_{j}\right)$ should be created in the reference image and the greyscale of $\mathrm{P}\left(x_{i}, y_{j}\right)$ should be represented by the pixel greyscale distribution in the template window $\mathrm{W}$. The subimage which is covered by the template window $\mathrm{W}$ in reference image should be set as T. Then in the matching image, the sliding window $\mathrm{W}^{\prime}$ of the same size as $\mathrm{W}$ centered the corresponding pixels P' $\left(x_{i}, y_{j}\right)$ should be created. The subimage which is covered by the window $\mathrm{W}^{\prime}$ in matching image should be set as $\mathrm{J}$. Finally, move the window $\mathrm{W}^{\prime}$ in the horizontal direction and the vertical direction with the distance of $\triangle \mathrm{x}$ and $\triangle \mathrm{y}$. If the matching region is the same, the difference of $\mathrm{T}$ and $\mathrm{J}$ is zero.

According to the theory of stereo vision and our equipements, the distance of $\triangle \mathrm{x}$ is zero for the eplipolar line and the distance of $\triangle \mathrm{y}$ is no more than Parallax d. It is shown in figure 2 for the practical situation of NCC. But Parallax $d$ is still large for image matching, so it is necessary to search for further more precise constraints.

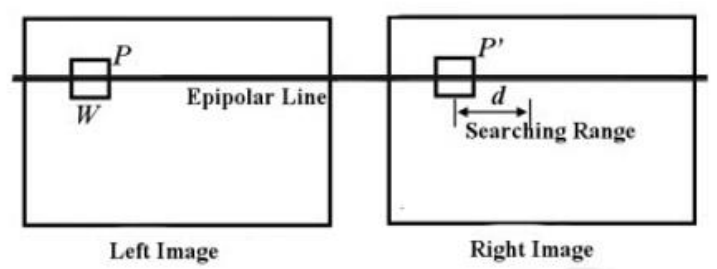

\section{Figure 2 Region Matching Image}

NCC algorithm is of high precision on gray scale smooth areas, fast computing speed. However, NCC algorithm has bad performance on repeat texture region and it is sensitive to the transformation of rotation, scale and projection. In our study, NCC algorithm has been used alone for flame image matching, but the result is poor.

\section{The Stereo Matching Algorithm for Flame based on Distance Ratio Constraint}

SIFT algorithm has the characteristic of invariance to the different projections which makes the feature points is stable. NCC algorithm has a good performance on gray scale smooth areas, but it fails to get the accurate result on the repeated texture area of flame image. In order to overcome abnormal gray points, the fuzzy boundary of flame and other disadvantages in flame image caused by the flicker and wobbling of dynamic flame and greatly improve the result of the flame image matching, a stereo matching algorithm for flame based on distance ratio constraint (DRCFMA) is proposed in this paper for flame based on distance ratio constraint, the sift operator and NCC algorithm. 
The core idea of DRCFMA is described in follows : First, extract the feature points of flame image by taking SIFT operator. Second, do the post-treatment to the point set above acquired including the method of Random Sample Consensus and calculating Euclidean distance ratio constraint of the corresponding points. Then the accurate feature matching point set is obtained. A new constraint of ratio of the transverse direction distance of controlled matching points has been integrated into NCC algorithm.

\subsection{The Post-treatment of SIFT Operation}

The feature points obtained are not always correct. There will be huge errors if these wrong feature points can't be removed. This paper adopts the Random Sample Consensus method (RANSAC) to eliminate the incorrect matched feature points.

The core idea of RANSAC is described as follows :

Set the initial optimal inner points $N_{i}$ as 0 . The steps of method as follows:

(1) Randomly select 4 pair initial matching points from I pair initial matching points (Any 3 points should not be on the same plane). The parameter $\mathrm{H}$ of projection transformation matrix between two planes could be calculated from linear computation of four group points.

(2) Calculate the distance of remaining (I-4) feature points between the coordinate of initial matching point and the coordinate changed by projection transformation matrix according to the (6).

$$
\mathrm{dv}=\mathrm{d}\left(\mathrm{A}_{i}^{\prime}, \mathrm{H} A_{i}\right)^{2}
$$

When distance, $d v$, is less than the threshold value $\mathrm{T} 1$, this feature point is the inner point; Otherwise, this feature point is the exterior point.

(3) Count the number of the inner point. If the number is greater than $N_{i}$, update $N_{i}$ as the new number of the inner point.

(4) After repeating above three steps some times (10000 times are set in our experiments), the number of inner point should remain unchanged. Select the transformation matrix with the most inner points and the minimum error function as the projection transformation matrix, so the transformation matrix has been very accurate at this time. Eliminate the matching pairs which has more errors than the threshold value. Then repeat the above steps $m$ times ( 3 times are set in experiments).

After RANSAC, feature vector is used as the further step for eliminating erroneously matched positions. In view that there is a huge difference of greyscale between the area inside the flame and the area outside the flame, the area inside the flame is the even grayscale areas and erroneously matched positions can be most likely presented in the edge of flame after SIFT, the Euclidean distance (7) of feature vector is introduced as the similarity measurement of feature points in different images.

$$
\mathrm{D}=\sqrt{\sum_{k=0}^{n}\left(X_{i}-X_{j}\right)^{2}}
$$

where $X_{i}$ refers the feature vector of ith feature point in the reference image, $X_{j}$ refers the feature vector of $j$ th feature point in the matching image. $\mathrm{D}$ is the Euclidean distance. Calculate the Euclidean distance of the matched feature points in two images and the ratio of the minimum and the maximum distance

$$
\mathrm{R}=\mathrm{Dmin} / \mathrm{Dscn}
$$

When $\mathrm{R}$ is less than the threshold value T2 (T2 is set as 0.9 in our following experiments), this feature point is erroneously matched; The greater the threshold value of T2 is, the less but more stable matching points will be obtained.

After above methods, matched feature points for constraint should be obtained. 


\subsection{The Constraint Ratio of Feature Matched Points}

In order to get the constraint ratio at the same epipolar line, a rectangular coordinate system ( $\mathrm{x}, \mathrm{y})$ should be established in pixels and the upper left corner point is the original point. Record the coordinate of matched feature points.

(1)If there are s pair matched points at the same epipolar line which means the matched points share the same Y coordinate. Set the $\mathrm{X}$ coordinate of ath matched point in left image and right image as $x_{l a} 、 x_{r a}$ and record the constraint ratio $P_{y a}$ which is defined as (9).

$$
P_{y a}=\frac{x_{l a}}{x_{r a}}
$$

Calculate the arithmetic mean value of the whole $P_{y a}$. The final constraint ratio of the epipolar line is defined as $(10)$

$$
P_{y a}(\text { mean })=\frac{\sum_{a=0}^{s} P_{y a}}{s}
$$

(2) If there is only one pair of matched points at the same epipolar line, (9) is used for this situation.

(3) If there is no matched points at the same epipolar line, the ratio of adjacent epipolar line should be referenced. The constraint ratio at this epipolar line is defined as (11).

$$
P_{y a}(\text { mean })=\frac{P(\mathrm{y}-1) a(\text { mean })+P_{(y+1) a}(\text { mean })}{2}
$$

\subsection{The Core Idea of Stereo Matching Algorithm for Flame based on Distance Ratio Constraint}

In the process of stereo matching, cross correlation coefficient of the template window in reference image and the corresponding widow in matching image $\mathrm{R}(\mathrm{u}, \mathrm{v})$ should be calculated as (12) when the corresponding window is sliding.

$$
\mathrm{R}(u, v)=\frac{\sum_{i=1}^{N 1} \sum_{j=1}^{N 2}\left(x_{i+u, j+v} \cdot y_{i j}\right)}{\left[\sum_{i=1}^{N 1} \sum_{j=1}^{N 2}\left(x_{i+u, j+v}^{2}\right)\right]^{1 / 2}\left[\sum_{i=1}^{N 1} \sum_{j=1}^{N 2}\left(y_{i j}^{2}\right)\right]^{1 / 2}}
$$

where $\mathrm{R}(\mathrm{u}, \mathrm{v})$ is the cross correlation coefficient of the location $(\mathrm{u}, \mathrm{v})$, where N1*N2 is the size of template window, where $x_{i+u, j+v}, y_{i j}$ is the greyscale of the location $(\mathrm{i}, \mathrm{j})$ in the reference image and $(i+u, j+v)$ in the matching image respectively.

Record the x-coordinate $x_{l w 1}$ of left edge of the template window and the xcoordinate $x_{r w 1}$ of the corresponding left edge of the matching window. Calculate the ratio $\mathrm{Wy} 1$ as (13).

$$
\mathrm{Wy} 1=\frac{x_{l w 1}}{x r w 1}
$$

Record the $\mathrm{x}$-coordinate $x_{l w 2}$ of right edge of the template window and the $\mathrm{x}$ coordinate $x_{r w 2}$ of the corresponding left edge of the matching window. Calculate the ratio Wy2 as (14).

$$
\mathrm{Wy} 2=\frac{x_{l w 2}}{x r w 2}
$$

When the matching window is sliding, the constraint ratio of feature matched points ratio should meet the requirement (15).

$$
\mathrm{Wy} 1<P_{y s}<\mathrm{Wy} 2
$$

Where $P_{y s}$ is the element in (10) and (11), Wy1 is the element in (13), Wy2 is the element in (14).

Record the x-coordinate $x_{l \mathrm{mcp}}$ of the center point of the template window and the xcoordinate $x_{r \mathrm{rcp}}$ of the center point of the corresponding matching window. Calculate the ratio $\mathrm{MCP}_{y}$ as (16).

$$
\mathrm{MCP}_{y}=\frac{x_{l \mathrm{mcp}}}{x_{r \mathrm{mcp}}}
$$

Here a threshold value TC should be given. 
When $\mathrm{TC}=\frac{\mathrm{MCP}_{y}}{P_{y s}}<1$, the $\mathrm{x}$-coordinate of key point in reference image is less than the $\mathrm{x}$ coordinate of the matching point in matched image.

When $\mathrm{TC}=\frac{\mathrm{MCP}_{y}}{P_{y s}}=1$, the $\mathrm{x}$-coordinate of key point in reference image is equal to the $\mathrm{x}$ coordinate of the matching point in matched image.

When $\mathrm{TC}=\frac{\mathrm{MCP}}{P_{y s}}>1$, the $\mathrm{x}$-coordinate of key point in reference image is greater than the $\mathrm{x}$-coordinate of the matching point in matched image.

TC is related to the accuracy requirement, Parallax $d$ and the size $m * n$ of template window.

In this paper, $\mathrm{TC}$ is constrained as (17)

$$
1-\frac{2 m}{d} \leq \mathrm{TC} \leq 1+\frac{2 m}{d}
$$

If the selected optimal greyscale matching point $R_{f i r}(\mathrm{u}, \mathrm{v})$ in matching image cannot meet the requirement of (15) and (17), the second optimal greyscale matching point $R_{s e c}(\mathrm{u}, \mathrm{v})$ should be selected, and so on. If the template window sliding into the bottom right corner, then the algorithm exits.

\subsection{The Stereo Matching Algorithm forFlame based on Distance Ratio Constraint}

Based on the above ideas, this paper presents a stereo matching algorithm for flame based on distance ratio constraint (DRCFMA). The result of DRCFMA could be used in the 3-D reconstruction of industrial flame. The detail of this algorithm is shown as follows:

Algorithm: stereo matching algorithm for flame based on distance ratio constraint (DRCFMA)

Step 1 : Get the preliminary matching feature points set of flame image by taking SIFT operator.

Step 2 : Do the post-treatment to the point set. Get the constraint points.

Step2.1 : RANSAC should be taken to eliminate the abnormal greyscale point.

Step2.2 : Euclidean distance ratio of the matched points should be calculated in order to eliminate the erroneously matched positions caused by the repeated texture area.

Step 3 : Calculate the constraint ratio by (10) and (11) .

Step $4:$ In the process of stereo matching, the optimum similarity of flame images should be obtained by (12). When (15)and (17) is satisfied, the matching area is valid. Otherwise the second optimum similar area should be selected for matching.

Step 5 : Repeat Step 3 and 4, until the template window moved into the lower right corner.

\section{The Experiment and Results}

With the help of Professor YanYong at the University of Kent, a set of images about industrial flame at a certain moment from eight perspectives had been obtained by the experimental equipment which is introduced by the section 2. To evaluate the performance of DRCFMA, a program about DRCFMA was coded on Visual C ++2010 platform.

\subsection{The Test Image}

Using the image capturing device introduced in the Section 2, the industrial turbulent flame images which are 8-bit greyscale digital images with the resolution of $256^{*} 256$ from eight perspectives $\left(0^{\circ} 、 22.5^{\circ} 、 45^{\circ} 、 67.5^{\circ} 、 90^{\circ} 、 112.5^{\circ} 、 135^{\circ} 、 157.5^{\circ}\right)$ had 
been synchronized obtained at a certain moment. The follow-up experiments are based on the following images in Fig.3.
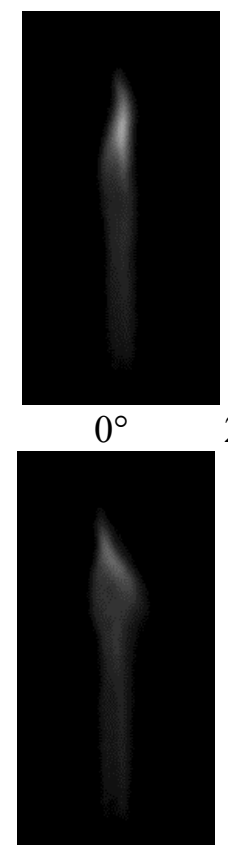

$90^{\circ}$

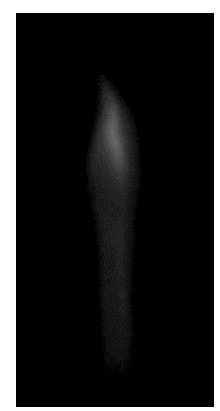

$22.5^{\circ}$

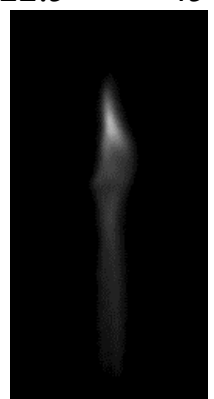

$112.5^{\circ}$

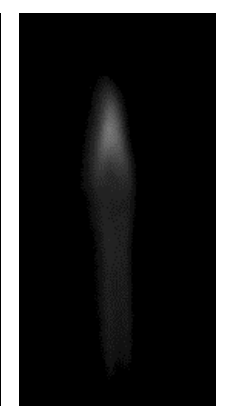

$67.5^{\circ}$

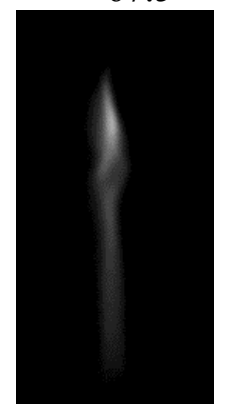

$157.5^{\circ}$
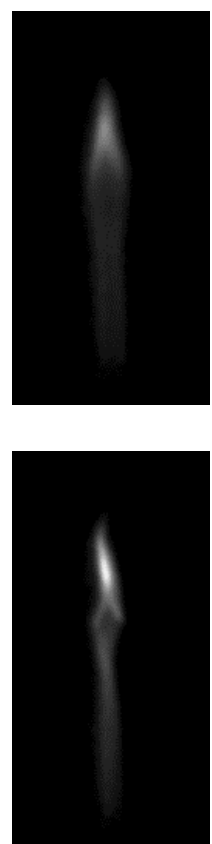

Figure 3. Flame Images Captured

\subsection{The Validation of the Match}

To verify the correctness and matching effect of DRCFMA, the experiments are done based on the above flame images. On each image, a number of true feature points have been chosen according to the characteristics of the turbulent flame. And the same time, a number of feature match points have been selected by DRCFMA. Due to the space limitations of the paper, only the image matching results of the angle of $0^{\circ}$ and $67.5^{\circ}$ and $157.5^{\circ}$ are given. The image of true feature points and the image of feature points selected by DRCFMA are given as a pair of images in the same angle in figure 4 . The red dots of the left side of image pairs represent the feature points selected by the selecting algorithm of feature points in the 3-D reconstruction system for industry flame developed by our research group according to the characteristics of industrial flame. The red dots of the right side of image pairs represent the feature points selected by DRCFMA.

As the feature points are selected in the pixel level, red dot is just a mark of feature points. Some red dots maybe represent the collections of a set of feature points which are located in close proximity. All the image matching results of 8 groups are given in table 1 . From table 1, we can find that the matching accuracy of 8 group images of the turbulent flame is remaining stable between $86 \%$ and $89 \%$. 


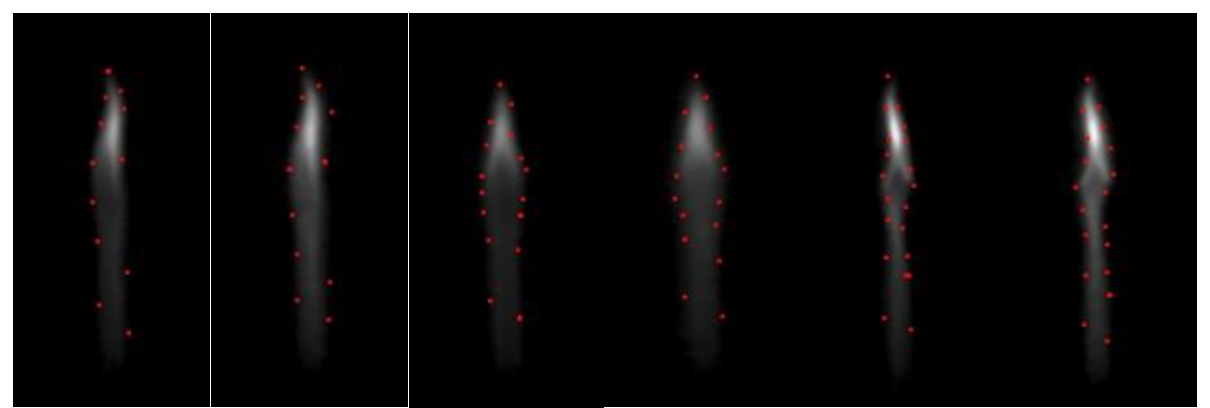

$\begin{array}{lll}\text { (a) } 0^{\circ} \text { matching result } & \text { (b) } 67.5^{\circ} \text { matching result } & \text { (c) } 157.5^{\circ} \text { matching result }\end{array}$

Figure 3. The Feature Point Matching Result of $0^{\circ}, 67.5^{\circ}$ and $157.5^{\circ}$

Table 1. The Feature Point Matching Result from 8 Projections

\begin{tabular}{|c|c|c|c|c|}
\hline Angle & $\begin{array}{c}\text { the number of } \\
\text { matched feature } \\
\text { points }\end{array}$ & $\begin{array}{c}\text { the number of matched } \\
\text { feature points correctly } \\
\text { detected }\end{array}$ & Accuracy & $\begin{array}{c}\text { Finished } \\
\text { Time }\end{array}$ \\
\hline $0^{\circ}$ & 47 & 42 & $89.3 \%$ & $105.3 \mathrm{~ms}$ \\
\hline $22.5^{\circ}$ & 45 & 40 & $88.89 \%$ & $105.4 \mathrm{~ms}$ \\
\hline $45^{\circ}$ & 46 & 40 & $86.96 \%$ & $105.4 \mathrm{~ms}$ \\
\hline $67.5^{\circ}$ & 47 & 42 & $89.36 \%$ & $105.3 \mathrm{~ms}$ \\
\hline $90^{\circ}$ & 45 & 40 & $88.89 \%$ & $105.4 \mathrm{~ms}$ \\
\hline $112.5^{\circ}$ & 46 & 41 & $89.13 \%$ & $105.3 \mathrm{~ms}$ \\
\hline $135^{\circ}$ & 46 & 41 & $89.13 \%$ & $105.3 \mathrm{~ms}$ \\
\hline $157.5^{\circ}$ & 47 & 42 & $89.36 \%$ & $105.3 \mathrm{~ms}$ \\
\hline
\end{tabular}

The accuracy and finished time of feature points matching in different projections are directly shown by Figure 4 and figure 5 as follows.

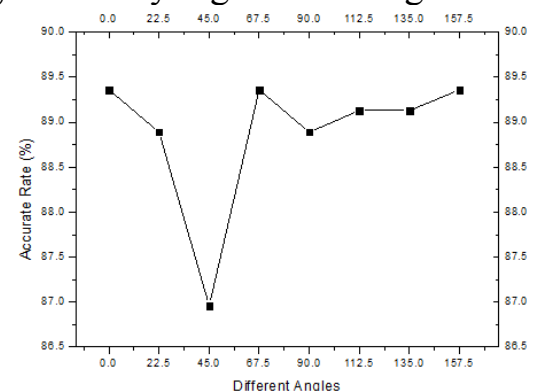

Figure 4. The Matching Accuracy

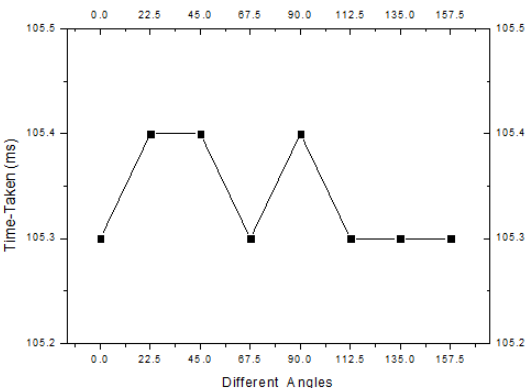

Figure 5. The Finished Time

There is a certain random variability of the matching result caused by flame images obtained from different projections. In the figure 4 and 5, the matching results of the flame images with $0^{\circ}, 67.5^{\circ}$ and $157.5^{\circ}$ show the high precision and short time-taken, because these images are obviously by greyscale layers and with more distinct edge. While the matching result of $45^{\circ}$ flame image is of a lower accuracy and longer timetaken, because its available information is less for the blurred edge and the smoothness of the gray value. In view of the overall performance of DRCFMA, the matching accuracy is over $86.5 \%$ and the finished time are within $105.4 \mathrm{~ms}$ in different projections. Through these experiments, DRCFMA has a good stability and high matching accuracy. Real-time performance also meets the requirement of industrial flame monitoring.

To verify the matching performance of DRCFMA, other feature points matching algorithms are compared with DRCFMA. As the current research to industrial flame is 
limited, there is few feature matching algorithm for flame in the literatures. Three mainstream algorithms (SIFT, Harris and SUSAN) for matching to scenery are selected to compare with DRCFMA. As the limit of length in this paper, the matching results of four algorithms on 8-bit greyscale digital image with zero angle are given as figure 6 . The red dots marks the feature points selected by the different matching algorithms. The total matching results for the eight projections are shown in Table 2.

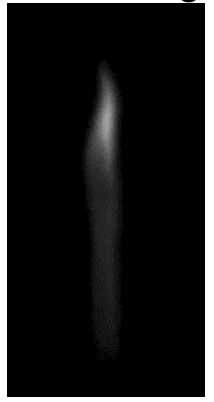

(1) $0^{\circ}$ original image

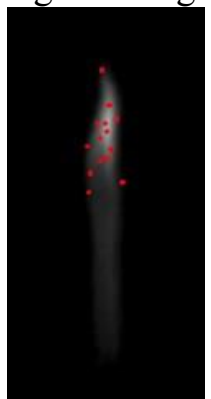

(4) result of SUSAN

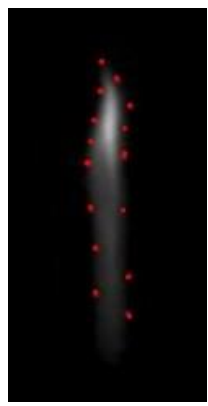

(2) $0^{\circ}$ image with true feature point

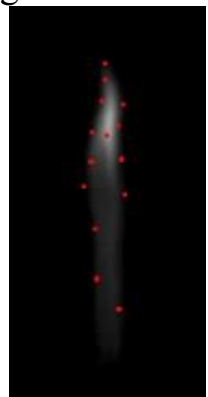

(5) result of HARRIS

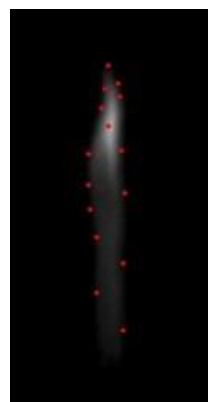

(3) result of SIFT

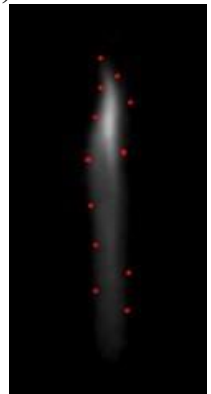

(6) result of DRCFMA

Figure 6. Matching Results of the Four Algorithms SIFT, HARRIS and DRCFMA

Table 2. The Total Matching Result of the Four Algorithms

\begin{tabular}{|c|c|c|c|c|}
\hline & $\begin{array}{c}\text { Total number of } \\
\text { matched feature } \\
\text { points }\end{array}$ & $\begin{array}{c}\text { Total number of } \\
\text { feature points } \\
\text { correctly matched }\end{array}$ & $\begin{array}{c}\text { Average } \\
\text { matching } \\
\text { accuracy }(\%)\end{array}$ & $\begin{array}{c}\text { Maximum of } \\
\text { finished time } \\
(\mathrm{ms})\end{array}$ \\
\hline SIFT & 378 & 326 & 86.24 & 110.2 \\
\hline SUSAN & 372 & 317 & 85.22 & 108.5 \\
\hline HARRIS & 373 & 321 & 86.06 & 107.7 \\
\hline DRCFMA & 369 & 328 & 88.89 & 105.4 \\
\hline
\end{tabular}

To further compare the performance of the four algorithms, two evaluation criteria were applied. One is the matching accuracy of feature points. The other is the maximum matching finished time. In order to guarantee the rationality of the experiment, the image of the same projection should be processed by the same algorithms 10 times and the average matching accuracy and the maximum finished time should be recorded.

Figure 6 and table 2 shows the accuracy of Harris is $86.06 \%$. Harris cannot get the desired matching result of the smoothness area in the lower part of the flame image. The reason is that the greyscale in vertical direction of flame image changes flatly. Once the greyscale change value is less than the value of threshold of Harris detection, Harris become invalid in that area. The failed matched feature points rate of the lower part of the flame image is $66.7 \%$. Susan is also not desirable for its matching accuracy of $85.22 \%$. From the results, Susan works poor at the blurred flame edges. The blurred flame edge is caused by the flame flicker. Either flame flicker or blurred flame edge will pose a disturbing effect on Susan. SIFT algorithm has the characteristic of invariance to the 
different projections which makes its accuracy is $86.24 \%$. But there are some similar areas in flame image which pose a disturbing effect on SIFT. The maximum time-taken is $110.2 \mathrm{~ms}$, more than other algorithms. Because of the fully consideration of the flame characteristics, DRCFM is efficient for its average matching accuracy of $88.89 \%$ and maximum time-taken of $105.4 \mathrm{~ms}$. DRCFM is better than the similar algorithms in the feature point matching of industrial turbulent flame images.

\subsection{The Result of 3-D Reconstruction of Flame}

Based on the flame images from eight projections, the matching result of DRCFMA is used for 3-D reconstruction of turbulent flame. As it is impossible to display 3-D flame field in paper, a set of depth maps separated from the 3-D reconstruction of flame field are given to demonstrate the result of the 3-D reconstruction for flame field in figure 7 . In this map set, the longitudinal depth of the two adjacent maps is $1.50 \mathrm{~mm}$.
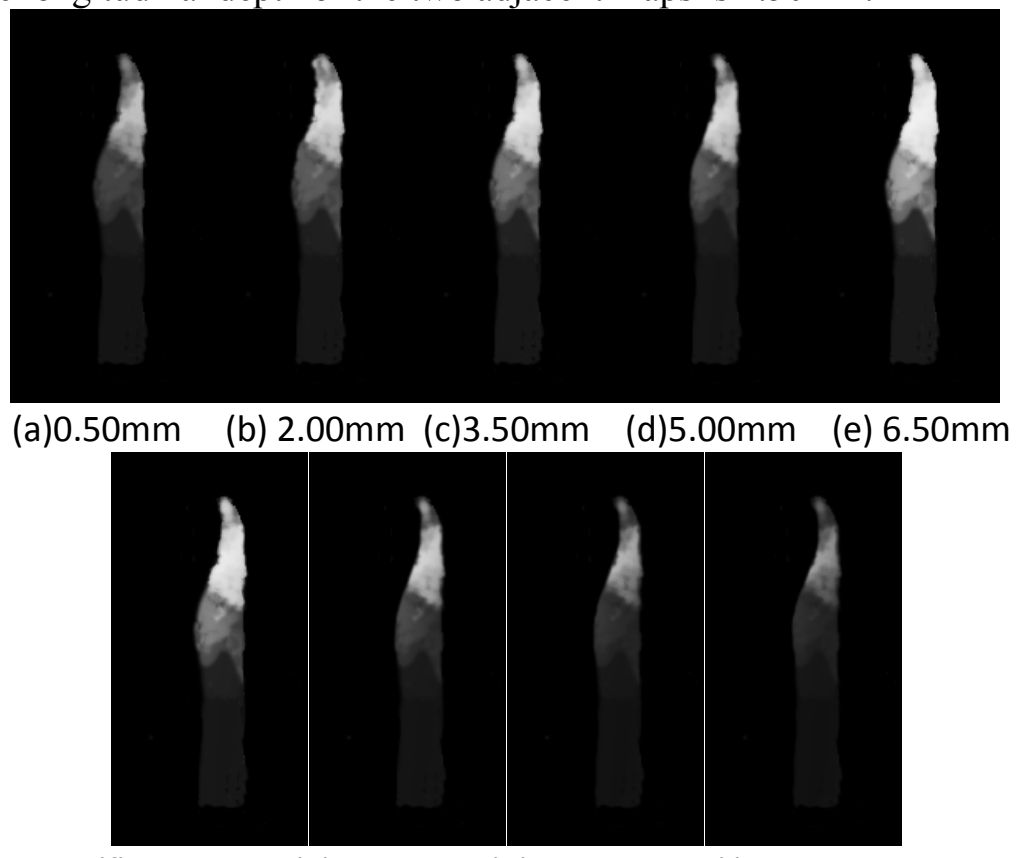

$\begin{array}{llll}\text { (f) } 8.00 \mathrm{~mm} & \text { (g) } 9.50 \mathrm{~mm} & \text { (h) } 11.00 \mathrm{~mm} & \text { (i) } 12.50 \mathrm{~mm}\end{array}$

\section{Figure 7. A Set of Depth Maps of 3-D Reconstruction of Flame Field}

In the figure 7, as the longitudinal depth increases, the depth map from (a) to (e) shows the scene that approached from the boundary to the center of flame. The depth map from (f) to (i) shows that after the longitudinal depth reached the radius of flame cross section, as the longitudinal depth increases, the scene that approached from the center to the other side of flame.

As is shown in figure 7, (b) is little more brighter and fuller in shape. (e) represents the greyscale image of flame center. (e) is also the image with the strongest brightness. (f) is little more dark than (e). That the flame cross-section area is decreasing from (e) to (i) shows the flame characteristic that the center flame cross-section is more fuller than the outside flame cross-section. Take the example of (d), four different greyscale layers are observed in top-down approach. The center is the brightest. The top is the second brightest. The bottom is the darkest. The value of greyscale is related with the flame temperature. The greater the value of greyscale is, the higher the temperature is. The center of flame is of the highest temperature.

From figure 7, the follow-up 3-D reconstruction maintain the undamaged flame characteristics. The internal structures and variations in luminous properties are clear in the reconstructed cross sections of the flame along the burner axis and radial directions. 
The boundary of 3-D reconstruction of flame is smooth without burr. DRCFMA has an excellent performance on fast reconstructing for 3-D flame field, which could help the further study on the internal structures analysis of flame and industrial flame control.

\section{Conclusion}

DRCFMA has been proposed and evaluated in this paper based on the characteristics of flame. Results have demonstrated that DRCFMA is efficient to matching the feature point with the high accuracy and short time. The follow-up 3-D reconstruction experiments have clear and continuously changing greyscale layers and undamaged flame characteristics by using the matching result of DRCFMA.

\section{Acknowledgement}

This study is supported by "the Fundamental Research Funds for the Central Universities (11MG11)". The professor YanYong in the university of Kent has put forward valuable suggestions to this paper. Thank professor YanYong and his team for providing us laboratory equipment and helping us to obtain the industrial turbulent flame image set at the same time from multiple projections.

\section{References}

[1] M.M. Hossain, G. Lu, D. Sun and Y. Yan, "Three-dimensional reconstruction of flame temperature and emissivity distribution using optical tomographic and two-colour pyrometric techniques", Measurement Science and Technology, vol.24, no.7, (2013).

[2] M.M. Hossain, G. Lu, and Y. Yan, "Three-Dimensional Reconstruction of Combustion Flames through Optical Fiber Sensing and CCD Imaging", IEEE International Instrumentation and Measurement Technology Conference (I2MTC 2011), (2011).

[3] S. M. Seitz, B. Curless, J. Diebel, D. Scharstein, and R. Szeliski, "A comparison and evaluation of multi-view stereo reconstruction algorithms". Proc. CVPR, (2006), pp. 519-528.

[4] D.G. Lowe, " Distinctive Image Features from Scale-Invariant Keypoints", International Journal of Computer Vision, vol. 60, no. 2, (2004), pp. 91-110.

[5] B. Cyganek and J.Borgosz, "A comparative study of performance and implementation of some areabased stereo algorithms", Proceedings of the $9^{\text {th }}$ International Conference on Computer Analysis of Images and Patterns, vol. 2014, (2001), pp. 709-716.

[6] M.A. Fischler, and R.C. Bolles, "Random Sample Consensus: A Paradigm for Model Fitting with Applications to Image Analysis and Automated Cartography", Communications of the ACM , vol. 24, no. 6, (1981), pp. 381-395.

[7] K. Mikolajczyk, C. Schmid, "An affine invariant interest point detector", European Conference of Comuter Vision, vol. 15, no. 3, (2002), pp. 128-130.

[8] H. Hirschmuller and D. Scharstein, "Evaluation of cost functions for stereo matching", Proc. of the International Conference on Computer Vision and Pattern Recognition, vol. 1, (2007), pp. 1-8. 